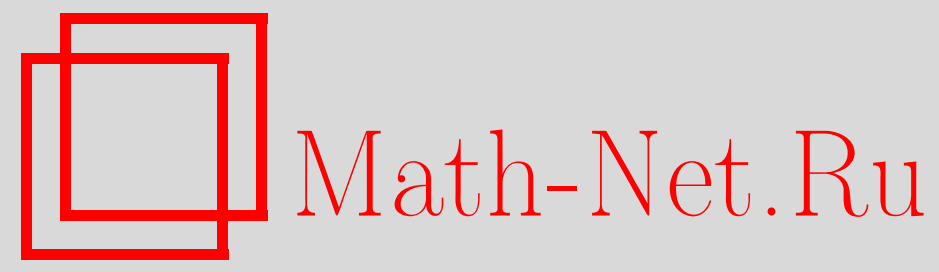

В. Г. Вовк, Чисто мартингальный вариант усиленного закона больших чисел Колмогорова, Теория вероятн. и ее примен., 1996, том 41, выпуск 3, 639-642

DOI: https://doi.org/10.4213/tvp3144

Использование Общероссийского математического портала Math-Net.Ru подразумевает, что вы прочитали и согласны с пользовательским соглашением http://www . mathnet.ru/rus/agreement

Параметры загрузки:

IP: 34.227 .88 .159

26 апреля 2023 г., 12:09:30

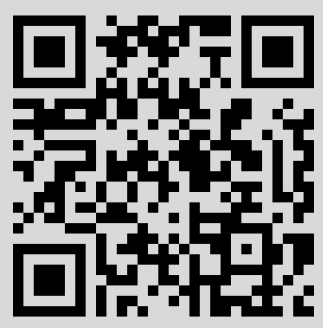


11. Шуреякое В. Н. Эргодические процессы Маркова. М.: Наука, 1989, 336 с.

12. Тутубалия B. Н. Теория вероятностей и случайных процессов. М.: Изд-во Московского ун-та, 1992, 395 с.

13. Невё Ж. Математические основы теории вероятностей. М.: Мир, 1969, 309 с.

14. Халмои II. Гильбертово пространство в задачах. М.: Мир, 1970, 352 с.

15. Гордия М. И., Лифиии Б. А. Центральная предельная теорема для стационарных процессов Маркова. - Докл. АН СССР, 1978, т. 239, № 4, с. 766-767.

Поступила в редакцию

23.III.1993

(C) $1996 \mathrm{r}$.

BOBK B. Г.*

\title{
ЧИСТО МАРТИНГАЛЬНЫЙ ВАРИАНТ УСИЛЕННОГО ЗАКОНА БОЛЬШИХ ЧИСЕЛ КОЛМОГОРОВА ${ }^{1)}$
}

\begin{abstract}
Рассматривается следующая альтернатива колмогоровской аксиоматике теории вероятностей: в качестве базисного выбирается понятие мартингала, а вероятности определяются при помоши мартингалов. В рамках этой «мартингальной» аксиоматики доказан «чисто мартингальный» вариант усиленного закона больших чисел Колмогорова, являющийся усилением обычного мартингального варианта.
\end{abstract}

Ключевые слова и фразы: мартингал, усиленный захон больших чисел, принцип невозможности системы игры.

1. Введение. Цель настоящей заметки - привлечь внимание к тому простому факту, что некоторые результаты теории вероятностей не требуют аксиоматики Колмогорова в полном объеме. Подробно будет рассмотрен только усиленный закон больших чисел (УЗБЧ) Колмогорова; некоторые другие результаты будут упомянуты в п. 5.

Под усиленным законом больших чисел Колмогорова будет пониматься следующее обобщение классического результата Колмогорова: если $\xi_{i}$ - мартингал-разность относительно фильтрации $\left(\mathcal{F}_{i}\right)$, то

$$
\sum_{i=1}^{\infty} \frac{\mathbf{E}\left(\xi_{i}^{2} \mid \mathcal{F}_{i-1}\right)}{i^{2}}<\infty \Longrightarrow \lim _{n \rightarrow \infty} \frac{1}{n} \sum_{i=1}^{n} \xi_{i}=0
$$

почти наверное. (Для любых двух событий $A$ и $B$ событие $A \Rightarrow B$ определяется как $A^{c} \cup B$; следовательно, «A $A B$ почти наверное» эквивалентно равенству нулю вероятности того, что $A$ выполнено, а $B$ - нет.) Фактически, это есть частный случай результата Чао (Chow) (см. [1, теорема 3.3.1]).

Формулировка усиленного закона больших чисел Колмогорова явно содержит только условные ожидания $\mathbf{E}\left(\xi_{i} \mid \mathcal{F}_{i-1}\right)$ (требуется их равенство нулю) и $\mathbf{E}\left(\xi_{i}^{2} \mid \mathcal{F}_{i-1}\right)$.

*Научный совет РАН по комплексной проблеме «Кибернетика», ул. Вавилова, 40, 117333 Москва, Россия.

1) Исследование поддержано Международным научным фондом Дж. Сороса, грант No MRS000. 
Однако из-за использования аксиоматики Колмогорова неявно требуется существование многих других вероятностных характеристик: например, мы вынуждены предполагать, что вероятность события $\left\{\xi_{1} \geqslant 0\right\}$ существует. Необходимость подобных допущений сужает область применимости усиленного закона больших чисел Колмогорова. Так, А. Н. Колмогоров отмечает в [2], что предположение о существовании вероятности для данного события является гипотезой, которая в каждом отдельном вопросе требует специальной проверки или обоснования.

В п, 2 описывается простая конструкция, которая позволяет сформулировать (см. п. 3) усиленный закон больших чисел Колмогорова в общем виде, без явно излишних допушений. Доказательства собраны в п. 4.

2. Нулевые событня. Для формулировки усиленного закона больших чисел требуется только определить понятие нулевого события (т.е. события вероятности 0); в п. 5 мы кратко обсудим общее понятие вероятности. Наше определение нулевого события будет основано на идее Дэйвида [3] (см. также [4, §3]).

Зафиксируем фильтрованное пространство $\left(\Omega,\left(\mathcal{F}_{0}, \mathcal{F}_{1}, \ldots\right), \mathcal{F}\right)$ с $\mathcal{F}_{0}=\{\varnothing, \Omega\}$. Обычно фильтрованное пространство дополняется до өероятностной модели добавлением вероятностной меры $P$ на $\mathcal{F}$. Если заранее выбранное событие $E \in \mathcal{F}$ удовлетворяет $P(E)=0$, мы можем быть (почти) уверены, что $E$ не произойдет (при условии, что мы принимаем модель). Это дает обычную интерпретацию усиленного закона больших чисел.

В этой заметке рассматривается другой тип моделей. Кокечномерная мартиягальная модель (нам потребуются только такие модели) получается из фильтрованного пространства $\left(\Omega,\left(\mathcal{F}_{0}, \mathcal{F}_{1}, \ldots\right), \mathcal{F}\right)$ добавлением стохастической последовательности $M$ со значениями в $\mathbf{R}^{k}$ (т.е. последовательности $M_{0}, M_{1}, \ldots$ таких случайных элементов со значениями в $\mathbf{R}^{k}$, что каждый $M_{n}$ является $\mathcal{F}_{n}$-измеримым), где $k-$ некоторое натуральное число; $M$ называется базисным мартияеалом. Предполагается, что $M_{0}=0$.

Из базисного мартингала можно получать другие мартингалы при помощи следующего известного приема. Если $V=\left(V_{1}, V_{2}, \ldots\right)$ - предсказуемая последовательность со значениями в $\mathbf{R}^{k}$ (т.е. каждое $V_{n}$ есть $\mathcal{F}_{n-1}$-измеримый случайный элемент со значениями в $\mathbf{R}^{k}$ ), то мартингальяое преобразование $V \cdot M$ определяется как стохастическая последовательность $(V \cdot M)_{n}=\sum_{i=1}^{n} V_{i} \cdot\left(M_{i}-M_{i-1}\right), n=0,1, \ldots$ $\left(\cdot\right.$ в правой части обозначает скалярное произведение векторов на $\left.\mathbf{R}^{k}\right)$. Стохастические последовательности вида $c+V \cdot M$, где $c \in \mathbf{R}$, называются $M$-мартингалами. (Игровая интерпретация этих определений изложена, например, в $[1, \S 2.9]$ ).

Событие $E \in \mathcal{F}$ назовем $M$-мулевым, если существует такой неотрицательный $M$-мартингал $S$, что $S_{0}=1$ и $S_{n}(\omega) \rightarrow \infty$ (при $n \rightarrow \infty$ ) для всех $\omega \in E$. Ннтерпретация этого определения дается следующим «инфинитарным принципом кевозможности системы игры». $[4, \S 3]$ : если $S$ - такой заранее выбранный неотрицательный $M$-мартингал, что $S_{0}=1$, то, пока мы принимаем модель, мы можем быть уверены, что $S_{n}(\omega)$ не будет стремиться к бесконечности (при $n \rightarrow \infty$ ), где $\omega$ - реализованный исход. (Принцип невозможности системы игры является мартингальным аналогом принципа «пренебрежения достаточно малыми вероятностями» - см. [5].) Таким образом, если $M$-нулевое событие выбрано заранее, мы можем быть уверены, что оно не прои зойдет.

Следующая теорема показывает, что определение $M$-нулевого события согласуется с аксиоматикой Колмогорова.

Теорема 1. Если $M-$ локальный мартинаал относительно вероятностяой меры $P$ ка $(\Omega, \mathcal{F})$ и событие $E \in \mathcal{F}$ является $M$-кулевым, то $P(E)=0$.

3. Основной результат. Будем говорить, что свойство $A(\omega)$ исхода $\omega \in \Omega$ выполнено $M$-почти маверное, если событие $\{\omega:$ не $A(\omega)\}$ является $M$-нулевым. Базисяая мартиягал-разяость определяется как стохастическая последовательность 
$M_{1}-M_{0}, M_{2}-M_{1}, \ldots$

Теорема 2. Пусть $\xi_{1}, \xi_{2}, \ldots-$ стохастическая последовательность $u d_{1}$, $d_{2}, \ldots$ - предсказуемая последовательность. Если базисной мартингал-разностью является $\left(\xi_{i}, \xi_{i}^{2}-d_{i}\right)_{i=1}^{\infty}$, то, M-почти наверное,

$$
\sum_{i=1}^{\infty} \frac{d_{i}}{i^{2}}<\infty \Rightarrow \lim _{n \rightarrow \infty} \frac{1}{n} \sum_{i=1}^{n} \xi_{i}=0 .
$$

Теорема 1 показывает, что теорема 2 влечет усиленный закон больших чисел Колмогорова.

4. Доказательства. Д о к а а т е ль с т о те о ре мы 1 . Пусть $E \in$ $\mathcal{F}$ есть $M$-нулевое событие. Существует такая предсказуемая последовательность $V$ со значениями в $\mathbf{R}^{k}$, что функция $S=1+V \cdot M$ неотрицательна и $S_{n}(\omega) \rightarrow \infty$ $(n \rightarrow \infty)$ для всех $\omega \in E$. Относительно $P$ функция $S$ является неотрицательным локальным мартингалом, и, следовательно, неотрицательным супермартингалом (по лемме Фату). Из неравенства Дуба для любой константы $c>0$ получаем $P(E) \leqslant$ $P\left\{\exists n: S_{n} \geqslant c\right\} \leqslant 1 / c ;$ следовательно, $P(E)=0$.

Доказательство теоремы 2 будет состоять из нескольких шагов (аналогичных шагам доказательства Липцера усиленного закона больших чисел Колмогорова см. [6, теорема VII. 5.4]). Вначале докажем аналог теоремы Дуба о сходимости мартингалов (применительно к неотрицательным мартингалам).

Лемма 1. Eсли $S_{n}$ - неотричательный $M$-мартиягал, mо $\lim _{n \rightarrow \infty} S_{n}$ существует $M$-почти наверное.

Д о к а з а т е л ь с т в о. Доказательство стандартно. Зафиксируем два положительных рациональных числа $a$ и $b, a<b$. Определим $\tau_{0}=0$ и, для $m=1,2, \ldots$,

$$
\sigma_{m}=\min \left\{i>\tau_{m-1}: S_{i}>b\right\}, \quad \tau_{m}=\min \left\{i>\sigma_{m}: S_{i}<a\right\} .
$$

Положим

$$
V_{i}=\left\{\begin{array}{ll}
1, & \text { если } \tau_{m-1}<i \leqslant \sigma_{m} \\
0 & \text { в противном случае }
\end{array} \text { дл некоторого } m,\right.
$$

и $S^{a, b}=S_{0}+V \cdot S(V \cdot S$ определяется точно так же, как $V \cdot M)$. Упорядочим все $S^{a, b}$ в последовательность $S^{1}, S^{2}, \ldots$ и положим $S^{0}=S$. Легко видеть, что $S_{n}^{*}=$ $\left(1 / S_{0}\right) \sum_{i=0}^{\infty} 2^{-i-1} S_{n}^{i}$ (мы предполагаем $S_{0}>0$ ) является таким неотрицательным $M$-мартингалом, что $S_{0}^{*}=1$ и $S_{n}^{*} \rightarrow \infty$, когда последовательность $S_{n}$ расходится.

Стохастическая последовательность вида $T_{n}=S_{n}+A_{n}$, где $S_{n}$ есть $M$-мартингал и $A_{n}$ - неубывающая предсказуемая последовательность с $A_{0}=0$, называется $M$-субмартингалом. Любую такую последовательность $A_{n}$ будем называть компенсатором субмартингала $T_{n}$.

Лемма 2. Если $T_{n}$ - неотрицательяьй $M$-субмартинаал с компенсатором $A_{n}$, то, $M$-почти наверное, $A_{\infty}<\infty \Rightarrow T_{n}$ сходится.

Д ок аз а т е ль с в о. Пусть $S_{n}=T_{n}-A_{n} ; S$ является $M$-мартингалом. Для $C=1,2, \ldots$ положим

$$
V_{n}^{C}=\left\{\begin{array}{ll}
1, & \text { если } A_{n} \leqslant C, \\
0 & \text { в противном случае, }
\end{array} \quad S_{n}^{C}=1+\frac{1}{C}\left(V^{C} \cdot S\right)_{n} .\right.
$$

Все $M$-мартингалы $S^{C}$ неотрицательны и удовлетворяют $S_{0}^{C}=1$. Легко видеть, что $M$-мартингал $R_{n}=\sum_{C=1}^{\infty} 2^{-C}\left(S^{C}\right)_{n}^{*}$ (где * обозначает преобразование из доказательства леммы 1 ) неотрицателен, $R_{0}=1$, и в случае, когда $T_{n}$ расходится и $A_{\infty}<\infty$, стремится к бесконечности.

Лемма 3. Если $S_{n}$ есть $M$-мартингал, $S_{n}^{2}-M$-субмартиягал и $A_{n}-$ компенсатор $M$ субмартинала $S_{n}^{2}$, то, $M$-почти наверное, $A_{\infty}<\infty \Rightarrow S_{n}$ cходится. 
Дока за тельст т о. Если $A_{n}$-компенсатор $M$-субмартингала $S_{n}^{2}$, то $A_{n}$ - также компенсатор $M$-субмартингала $\left(S_{n}+1\right)^{2}$. По лемме $2, S_{n}^{2}$ и $\left(S_{n}+1\right)^{2}$ сходятся при $A_{\infty}<\infty$ ( $M$-почти наверное). Остается заметить, что $S_{n}=\frac{1}{2}\left(\left(S_{n}+\right.\right.$ $\left.1)^{2}-S_{n}^{2}-1\right)$.

Д о к а з а т е л с с $\Upsilon$ в о т е о р е м ы 2. По лемме 3 (примененной к $M$-мартингал-разности $\left.\xi_{i} / i\right), \sum_{i=1}^{\infty} d_{i} / i^{2}<\infty \Rightarrow \sum_{i=1}^{\infty} \xi_{i} / i$ сходится, $M$-почти наверное. Остается применить лемму Кронекера (см., например, $[1$, лемма 3.2.3]), из которой следует, что для любой последовательности $x_{1}, x_{2}, \ldots$ действительных чисел выполнено $\sum_{i=1}^{\infty} x_{i} / i$ сходится $\Rightarrow \lim _{n \rightarrow \infty}(1 / n) \sum_{i=1}^{n} x_{i}=0$.

5. Замечания. В этой заметке мы определили только события вероятности 0 . Однако формулировка некоторых важных предельных теорем (например, центральной предельной теоремы) требует отличных от 0 вероятностей. Построение таких вероятностей, проведенное в $[4, \S 4]$, легко переносится на мартингальные модели. Определив отличные от 0 вероятности, мы можем, например, применить метод Линдеберга для дохазательства чисто мартингального варианта центральной предельной теоремы (аналогично доказательству теоремы 5 в [4]).

В этой заметке мы пытались изложить основную идею мартингального подхода к основаниям теории вероятностей на максимально простом примере. В частности, рассматривался только случай дискретного времени. Случаю непрерывного времени посвящена статья [7].

Может оказаться полезным рассматривать в качестве основного понятия не мартингал, а супермартингал (в этом случае мы должны ограничиться неотришательными последовательностями $V_{n}$ при определении «супермартингального преобразования»). В этой «супермартингальной схеме» теорема 2 утверждает, что (1) выполнено, если базисная супермартингалт-разность $-\left(\xi_{i},-\xi_{i}, \xi_{i}^{2}-d_{i}, d_{i}-\xi_{i}^{2}\right)_{i=1}^{\infty}$. Преимущество супермартингальной схемы проявляется в том, что для справедливости заключения теоремы 2 достаточно только потребовать, чтобы $\left(\xi_{i},-\xi_{i}, \xi_{i}^{2}-\right.$ $\left.d_{i}\right)_{i=1}^{\infty}$ было базисной супермартингал-разностью (единственное изменение в доказательстве - слова « $M$-мартингал» и «M-субмартингал» следует заменить на « $M$ супермартингал» и «семимартингал» соответственно).

\section{СПИСОК ЛИТЕРАТУРЫ}

1. Stout W. F. Almost sure convergence. New York: Academic Press, 1974, 381 p.

2. Колмогоров А. Н. Вероятность. - Большая Советская Эициклопедия, 3-е изд. Т. 4. М.: Советская Энциклопедия, 1971, с. 544.

3. Dawid A. P. Calibration-based empirical probability (with discussion). - Ann. Statist., 1985 , v. 13, p. 1251-1285.

4. Vovk V. G. A logic of probability, with application to the foundations of statistics (with discussion). - J. Roy. Statist. Soc., Ser. B, 1993, v. 55, p. 317-351.

5. Прохоров Ю. В., Севастьянов $B$. . А. Вероятностей теория. - Большая Советская Энциклопедия, 3-е изд. Т. 4. М.: Советская Энциклопедия, 1971, с. ‘540-543.

6. Ширяев A. Н. Вероятность, 2-е изд., перераб. и доп. М.: Наука, 1989, 640 с.

7. Vovk V. G. Forecasting point and continuous processes: prequential analysis. - Test, 1994, v. 2. 